\title{
Motivational Effects of Enhanced Expectancies for Motor Learning in Individuals With High and Low Self-Efficacy
}

\author{
Saeed Ghorbani' (D) and \\ Andreas Bund ${ }^{2}$
}

\begin{abstract}
Previous research has shown that enhanced expectancies can foster a person's motivational state and facilitate motor learning. However, the effects of enhanced expectancies on motivational state and subsequent motor learning in individuals with varied motivational states (e.g., self-efficacy; SE) are not well understood. This study examined the effects of enhanced expectancies on motivational state and motor learning in individuals with high and low SE by manipulating the type of knowledge of results (KR). We selected 60 participants from $25 \mathrm{I}$ male undergraduate students, based on their SE level, and assigned them to four groups: (a) Good Performance KR and High SE, (b) Poor Performance KR and High SE, (c) Good Performance KR and Low SE, and (d) Poor Performance KR and Low SE. We asked participants to throw beanbags at a target with their nondominant hand during an acquisition phase ( 10 blocks of six trials each) and during subsequent retention and transfer tests. During acquisition, the Good KR groups received KR from their three most accurate trials in each training block, whereas the Poor KR groups received KR from their three poorest attempts in each block. We measured accuracy scores and changes in $\mathrm{SE}$ as dependent variables. Results showed that KR from good trials, relative to KR
\end{abstract}

\footnotetext{
'Department of Physical Education and Sport Science, Aliabad Katoul Branch, Islamic Azad University, Aliabad Katoul, Iran

${ }^{2}$ Institute of Applied Educational Sciences, University of Luxembourg, Luxembourg

Corresponding Author:

Saeed Ghorbani, Department of Physical Education and Sport Science, Aliabad Katoul Branch, Islamic Azad University, Aliabad Katoul, Iran.

Email: s.ghorbani@aliabadiau.ac.ir
} 
from poor trials, led to better accuracy scores during acquisition and retention and transfer tests, independent of participants' initial SE levels. Moreover, KR on good trials enhanced SE in the Good KR and Low SE group and kept SE at a high level in the Good KR and High SE group during acquisition and on the retention and transfer tests. These results provide support for the OPTIMAL (optimizing performance through intrinsic motivation and attention for learning) theory of motor learning, and we discuss results in terms of the potential effects of positive feedback on motor performance in professional athletes.

\section{Keywords}

enhanced expectancies, self-efficacy, the OPTIMAL theory

\section{Introduction}

In recent years, research has shown that providing learners with positive performance feedback can enhance learners' expectancies and subsequently facilitate motor performance and learning (Ghorbani, 2019; Gonçalves, Cardozo, Valentini, \& Chiviacowsky, 2018; Lessa, Tani, \& Chiviacowsky, 2018; Saemi, Porter, Ghotbi-Varzaneh, Zarghami, \& Maleki, 2012; Saemi, Wulf, GhotbiVarzaneh, \& Zarghami, 2011). Enhancing learners' performance expectancies is considered a motivational factor in the OPTIMAL (optimizing performance through intrinsic motivation and attention for learning) theory of motor learning (Wulf \& Lewthwaite, 2016), whereby learners' positive expectancies are operationalized by the self-efficacy (SE) construct. SE is generally defined as the belief in one's ability to succeed in specific situations or to execute a task (Bandura, 1977).

A common research method for enhancing learners' motivational states is to provide them knowledge of results (KR) from their more accurate (i.e., good) trials versus their less accurate (i.e., poor) trials. KR from good trials has been shown to lead to higher SE beliefs and, thereafter, to more effective learning (Badami, VaezMousavi, Wulf, \& Namazizadeh, 2011, 2012; Saemi et al., 2011, 2012). For example, in a golf-putting task, Badami et al. $(2011,2012)$ found that KR from good trials led to significantly better motor learning and higher levels of perceived competence and self-confidence than did KR from poor trials. In addition, Saemi et al. $(2011,2012)$ demonstrated that KR from good trials led to higher SE and improved motor learning. Chiviacowsky and Wulf (2007) found no significant differences in throwing beanbags at a target with the nondominant hand during an acquisition phase, but, on delayed retention, KR from good trials resulted in significantly better performance than KR from poor trials. Chiviacowsky, Wulf, Wally, and Borges (2009) replicated these results with older adults. Accordingly, Wulf and Lewthwaite (2016) proposed that 
motivational factors mediate observed learning benefits following positive feedback (e.g., KR from good trials).

While previous research has identified this mediating role of enhanced motivational state associated with the benefits of positive performance KR on motor learning (Saemi et al., 2011, 2012; Wulf, Chiviacoswsky, \& Cardozo, 2014), these prior studies did not address how learners whose sense of SE varied might differentially respond to good or poor performance KR. Previous studies (Badami et al., 2011, 2012; Saemi et al., 2011, 2012) generally ignored the role of a priori differences in motivational state among individual participants. But, if motivational state mediates the relationship between positive performance feedback and motor learning, then a priori individual differences in motivational state must be considered. Therefore, this study sought to examine the good and poor performance KR effects on motivational state and motor learning in individuals who began motor learning with high versus low SE levels. We hypothesized that individuals with high and low SE would differ in their retention and transfer performance as a result of receiving good or poor performance KR. We used a $2 \times 2$ factorial design to compare individuals who reported either a high or a low level of SE and then randomly assigned them to good and poor performance KR, creating four experimental groups: (a) Good KR and High SE, (b) Poor KR and High SE, (c) Good KR and Low SE, and (d) Poor KR and Low SE. We used the motor learning task and procedure in studies by Chiviacowsky and Wulf (2007) and Chiviacowsky et al. (2009).

\section{Method}

\section{Participants}

This research protocol was developed and performed in accordance with the Declaration of Helsinki and was approved by the University's Institutional Review Board. Furthermore, all participants gave written informed consents. We tested a large sample $(N=251)$ of male undergraduate students (bachelor students of various disciplines who took part in first course of physical education) to determine their self-reported SE levels. All participants were right-handed and had no prior experience with the motor task. Before trying the motor task, participants rated themselves with respect to their expected performance at it - throwing a beanbag with the nondominant arm to hit a 100-point target on one of six trials. They used a rating scale ranging from 10 (not confident) to 100 (absolutely confident). We assigned students with low SE self-rating scores (between 10 and 20) to the Low SE group and students with high SE scores (between 90 and 100) to the High SE group, yielding a final participant sample of 60 male students ( $M$ age $=21.35$, $S D=1.86$ years), 30 in each SE group. We then randomly allocated these participants to separate groups that would receive either good performance KR or 
poor performance KR, creating four experimental groups: (a) Good KR and High SE $(n=15)$, (b) Poor KR and High SE $(n=15)$, (c) Good KR and Low SE $(n=15)$, and (d) Poor KR and Low SE $(n=15)$.

\section{Motor Task}

The motor task was similar to one used by Chiviacowsky and Wulf (2007) and Chiviacowsky et al. (2009). The participants were asked to throw beanbags with the nondominant arm at a target on the ground. The distance between the participant and the center of the target was three meters. Concentric circles around the target with radiuses of $10,20,30,40,50,60,70,80,90$, and $100 \mathrm{~cm}$ served to determine the accuracy of the throws. If the beanbag landed in the center of the target, then the score was 100. If it landed in one of the other circles, then the score was $90,80,70,60,50,40,30,20$, or 10 points, respectively. Finally, if it landed outside the target, we recorded a score of 0 (Chiviacowsky \& Wulf, 2007).

\section{Procedure}

The participants were tested individually on two consecutive days. Prior to data collection, participants were given general information about the experimental procedure and asked to complete a questionnaire seeking information such as age, laterality, and previous experiences with the motor task. Finally, the participants were given brief instructions about the beanbag-throwing task, which consisted of holding the beanbag with the nondominant hand and throwing it at the target. During the acquisition phase, participants performed 10 blocks of six trials each, and one day later, they completed the retention and transfer tests, consisting of 10 trials each without KR. In the transfer test, the distance to the target was changed from three to four meters. The participants were given six seconds to execute each throw. The participants were allowed to look at the target before each block, but during the acquisition, retention, and transfer phases, they were prevented from viewing the outcomes by wearing opaque swimming goggles. At the end of each acquisition block, participants in the Good KR and High SE and Good KR and Low SE groups received KR from their three most accurate trials in that block, whereas those in the Poor KR and High SE and Poor KR and Low SE groups received KR from their three poorest attempts in that block. All participants had been told that, at the end of each block, they would be given KR about three throws, but they had not been told whether the KR would refer to their best or worst attempts (Chiviacowsky \& Wulf, 2007). KRs from all three trials were written on a board and provided to the participant for 15 seconds. It included the number of attempts, the direction, and the score of the landing relative to the center of the target. That is, if the beanbag landed in the upper part of the target, then a 
plus sign was added to the throwing score (e.g., +50 ). Conversely, if the beanbag landed in the lower part of the target, then a minus sign was presented before the throwing score (e.g., -50 ). Therefore, KR included information about the throw's distance from the center of the target, as well as information about the direction of the error. We measured time with a digital chronometer. During the experimental procedure, prior to each acquisition block and before the retention and transfer tests, all participants completed the same SE scale that had been previously used to select the participants with high and low SE levels.

\section{Statistical Analysis}

We averaged accuracy scores in the first acquisition block across all six trials and analyzed baseline performance in a 2 (SE: High vs. Low $) \times 2($ KR: Good vs. Poor) analysis of variance (ANOVA). We further averaged accuracy scores in the acquisition phase across 10 blocks of six trials each in a 2 (SE: High vs. Low $) \times 2$ (KR: Good vs. Poor $) \times 10$ (Block) repeated measures analysis of covariance, using first block scores as a covariate. We averaged accuracy scores on the single block retention and transfer tests across all 10 trials and analyzed them in a 2 (SE: High vs. Low) $\times 2$ (KR: Good vs. Poor) analysis of covariance, using first block scores as a covariate. Initial (baseline) SE scores and SE scores on the retention and transfer tests were analyzed in a 2 (SE: High vs. Low $) \times 2$ (KR: Good vs. Poor) ANOVA. Finally, SE scores in the acquisition phase were analyzed in a 2 (SE: High vs. Low $) \times 2$ (KR: Good vs. Poor) $\times 10$ (Block) repeated measures ANOVA. When there were significant group differences, we calculated partial eta squared $\left(\eta^{2}\right)$ as the effect size. Moreover, we used simple linear regression analyses to determine possible associations between the SE and accuracy scores during acquisition and on the retention and transfer tests. First, we used the SE scores at baseline as predictors of performances in the first block, and we used the SE scores obtained before the retention and transfer tests as predictors of performance on the retention and transfer tests, respectively. Second, we used performance scores from the first block as predictors of SE in second block, and we used performance scores from the last block as predictors of SE taken before the retention test. We used the Shapiro-Wilk test to examine normality of accuracy scores at baseline. For all analyses, we set the statistical significance level at $p<.05$.

\section{Results}

\section{Accuracy Scores}

The results of the Shapiro-Wilk test showed that the accuracy scores at baseline were normally distributed. The analysis of the first block showed no significant 
main effects for $\mathrm{KR}$ or $\mathrm{SE}$, and there was no significant $\mathrm{KR} \times \mathrm{SE}$ interaction at baseline.

During the acquisition phase, there was a main effect for $\mathrm{KR}, F(1,55)=$ 51.99, $p \leq .001, \eta^{2}=.48$. Moreover, there was a significant Block $\times \mathrm{KR} \times \mathrm{SE}$ interaction, $F(8,440)=2.152, p<.05, \eta^{2}=.03$. However, the main effects of Block as well as SE were not significant. As shown in Figure 1, the Good KR groups achieved higher accuracy scores than the Poor KR groups. No other interactions were significant.

The data from the retention test indicated a main effect of $\mathrm{KR}, F(1,55)=20.03$, $p \leq .001, \eta^{2}=.26$, with the Good KR groups showing better throwing accuracy than the Poor KR groups. There was no significant main effect of SE and no significant interaction. On the transfer test, there were significant main effects for KR and SE, $F(1,55)=27.29, p \leq .001, \eta^{2}=.33$ and $F(1,55)=9.52, p \leq .01$, $\eta^{2}=.14$, respectively. There was no significant $\mathrm{KR} \times \mathrm{SE}$ interaction. With regard to KR, the Good KR groups had higher accuracy scores compared with the Poor KR groups. The main effect of SE occurred because of the higher accuracy scores of the performance of the Good KR and Low SE group (see Figure 1).

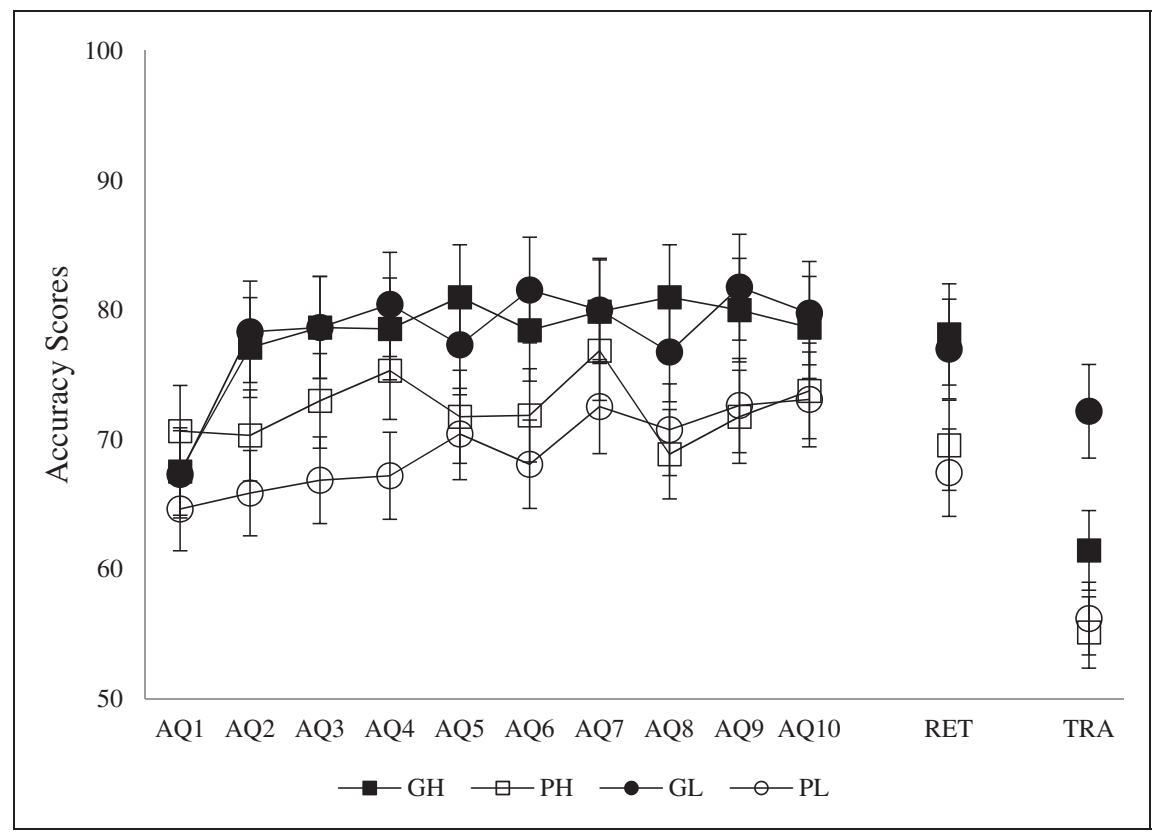

Figure I. Accuracy scores across the acquisition phase and on the retention and transfer tests for all groups. Note. GH $=$ Good KR and High SE; PH = Poor KR and High SE; $\mathrm{GL}=$ Good KR and Low SE; PL $=$ Poor KR and Low SE; AQ = acquisition phase; $\mathrm{RET}=$ retention test; TRA $=$ transfer test. 


\section{SE Scores}

An analysis of the first block showed no significant main effect for KR. However, the main effect for SE was statistically significant, $F(1,56)=3705.68, p \leq .001$, $\eta^{2}=.98$. The $\mathrm{KR} \times \mathrm{SE}$ interaction was not significant. Confirming the prestudy group assignment, participants in the High SE conditions reported much higher baseline SE scores than those in the Low SE conditions.

During the acquisition phase, there were significant main effects for Block, $F(9,504)=16.81, p \leq .001, \eta^{2}=.23$; for KR, $F(1,56)=103.28, p \leq .001, \eta^{2}=.64$; and for SE, $F(1,56)=124.42, p \leq .001, \eta^{2}=.69$. Moreover, all of the interactions including the Block $\times \mathrm{KR}$, Block $\times \mathrm{SE}, \mathrm{KR} \times \mathrm{SE}$, and Block $\times \mathrm{KR} \times \mathrm{SE}$ were significant (each $p \leq .01$ ). Here, participants in the Good KR groups had higher average SE scores than those in other groups. Moreover, participants in the Good KR and Low SE group showed increase in SE and those in the Good KR and High SE group showed no changes in SE. In addition, participants in the Poor KR and High SE group showed decrease in SE and those in the Poor KR and Low SE group showed a slight increase in SE. Overall, participants reported increases in their SE scores during the acquisition phase following exposure to the Good KR conditions and decreases in SE scores following exposure to the Poor KR conditions during the acquisition phase.

Finally, analyses of the results from retention and transfer tests demonstrated significant main effects for $\mathrm{KR}, F(1,56)=17.87, p \leq .001, \eta^{2}=.24$ and $F(1,56)=31.32, p \leq .001, \eta^{2}=.35$, respectively, and for SE, $F(1,56)=6.02$, $p=.017, \eta^{2}=.09$ and $F(1,56)=8.73, p=.005, \eta^{2}=.13$, respectively. No significant interactions were observed. On both tests, participants in the Good KR groups reported higher SE levels than those in the Poor KR groups. Moreover, participants in the High SE groups reported higher SE scores at retention and transfer than those in the Low SE groups; for example, the Good KR and High SE group had higher SE scores than the Good KR and Low SE group, and similarly, the Poor KR and High SE group had higher SE scores than the Poor KR and Low SE group.

\section{Regression Analyses}

SE scores at baseline did not significantly predict performances in the first block, but performance in first block significantly predicted SE before the second block, $F(1,58)=8.16, p=.006$, adjusted $R^{2}=.108, \beta=.351$. Performance in the last block also significantly predicted SE before the retention test, $F(1,58)=4.60, p=.036$, adjusted $R^{2}=.058, \beta=.271$. SE scores before the retention test significantly predicted performance on the retention test, $F(1,58)=11.44, p \leq .001$, adjusted $R^{2}=.15, \beta=.406$, and SE scores before the transfer test significantly predicted performance on the transfer test, $F(1,58)=8.22, p=.006$, adjusted $R^{2}=.109, \beta=.352$. 


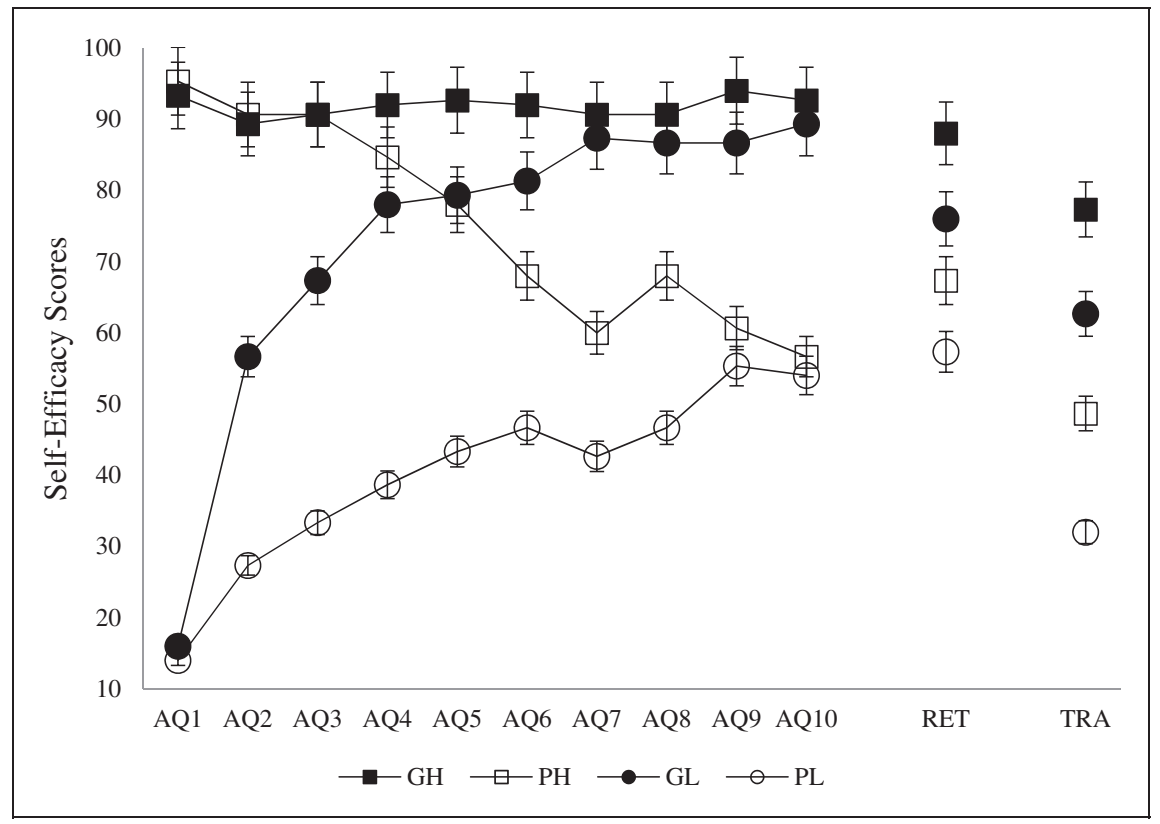

Figure 2. SE scores across the acquisition phase and on the retention and transfer tests for all groups. Note. HG = High SE and Good KR; HP = High SE and Poor KR, LG = Low SE and Good KR; $L P=$ Low SE and Poor KR; $A Q=$ acquisition phase; RET = retention test; TRA $=$ transfer test.

\section{Discussion}

Previous research has clearly demonstrated that KR from well-performed (good) trials versus KR from poorly performed (poor) trials enhances motivational state and motor learning in novice performers (Badami et al., 2011, 2012; Saemi et al., 2011, 2012). However, no research has yet investigated what role the learner's initial motivational state plays in responsiveness to KR type. This study was designed to investigate this issue. Our results replicated earlier findings that, during acquisition, KR from good trials resulted in significantly better performance accuracy than KR from poor trials. Similarly, KR from good trials, relative to KR from poor trials, led to significantly better accuracy on the retention test. Participants' initial SE levels had no mediating influence on this beneficial effect of positive feedback on motor performance and learning on acquisition and the retention test. However, on the transfer test, the Good KR and Low SE group had higher accuracy scores than the other three groups. These results are in line with previous studies in which enhanced expectancies benefitted motor performance and motor learning (Ávila, Chiviacowsky, Wulf, 
\& Lewthwaite, 2012; Badami et al., 2012; Chiviacowsky \& Wulf, 2007; Chiviacowsky et al., 2009; Ghorbani, 2019; Gonçalves et al., 2018; Grand, Daou, Lohse, \& Miller, 2017; Lessa et al., 2018; Montes, Wulf, \& Navalta, 2018; Ong \& Hodges, 2018; Pascua, Wulf, \& Lewthwaite, 2015; Saemi et al., 2011, 2012; Stoate, Wulf, \& Lewthwaite, 2012; Wulf et al., 2014), but they extend these findings to suggest a particular benefit for individuals with low SE ratings.

In addition, our results showed that during acquisition, KR from good trials did not (further) increase SE of participants in Good KR and High SE group (see Figure 2), as these participants simply maintained their initially high level of SE during acquisition. On the other hand, KR from good trials significantly enhanced SE for participants in the Good KR and Low SE group during acquisition. During the second half of the acquisition phase (i.e., Blocks 6-10), these low SE learners showed a similar SE level to learners in the Good KR and High SE group (see Figure 2). Both of these Good KR groups reported higher SE on the retention and transfer tests than the Poor KR groups. These results are in line with previous research showing good KR benefits (Badami et al., 2011, 2012; Saemi et al., 2011, 2012) and provide support for the OPTIMAL theory of motor learning (Wulf \& Lewthwaite, 2016), positing that KR from good trials leads to increases in motivational state that might partially explain better motor learning. Moreover, our results revealed that, for learners who reported high baseline SE (presumably indicating high motivation), KR from good trials was associated with continued high self-reported SE. This finding has important implications for the role of positive feedback on the improved motor performance of professional athletes with high levels of SE.

OPTIMAL theorists assume that enhanced expectancies facilitate motor learning by making dopamine available for memory consolidation and neural pathway development, thus contributing to efficient goal-action coupling by preparing the motor system for task execution (Wulf \& Lewthwaite, 2016). In this way, the enhanced expectations of our low SE participants and the maintained high expectancies of our high SE participants following good performance KR may have led to more effective retention and transfer performance.

Of note, we found that KR from poor trials was associated with decreased SE during acquisition for participants in the Poor KR and High SE group. These findings are in line with previous research (Saemi et al., 2012) showing that poor performance reduces SE for individuals with high baseline SE, perhaps explaining the lower performance of this group on the retention test. Surprisingly, KR from poor trials slightly increased SE during acquisition in the Poor KR and Low SE group, possibly because the specific data in their poor performance KR showed them to be performing slightly better than the severely poor performance they expected, as indicated by the nature of our SE rating scale. At the beginning of the study, these learners rated their SE as 10 or 20, 
while their "poor" performance KR indicated that poor performance beanbag throws were close to the scores from good trials (e.g., 50, 60). This might have inspired the learners in this group to increase their confidence ratings in comparison to their very low SE scores at the beginning of the study. Interestingly, at the end of the acquisition phase, there were no significant differences in the SE scores of the two Poor KR groups, reflecting the significant gains of the low SE participants, even when exposed to this Poor KR condition.

According to the regression analysis, there were reciprocal effects between participants' performance and SE. Performance in the first and last acquisition blocks predicted SE scores in the second block and before the retention test, respectively, and SE scores just before the retention and transfer tests predicted performance on these tests. These results are in accordance with Wulf et al.'s (2014) results and provide further support for predictions from the OPTIMAL theory of motor learning (Wulf \& Lewthwaite, 2016), again showing that enhanced expectancies mediated motor learning KR benefits.

Among this study's limitations, we used participants' SE as both an independent variable (with which to select participants) and a dependent variable (to measure the effects of positive feedback on motivational state). However, because we aimed to study how learners with variant SE responded motivationally to positive or negative KR, we believe the double function of SE to be acceptable in this case. Nevertheless, future studies might operationalize participants' a priori motivational state not only by SE but also by using other cognitivemotivational variables such as expectancies, goal-orientation, and self-concept. Such an approach would provide additional information about other learner traits. Second, we used only accuracy scores to measure motor performance and learning; future researchers might measure other variables such as movement form or kinematic and kinetic variables.

In conclusion, this study replicated prior findings that KR from good trials facilitated motor learning and further showed this to be true for both individuals with high and low a priori SE. We further showed that KR from good trials was associated with particularly enhanced motivational states of individuals with low self-reported SE at the beginning of the study, while individuals with high SE maintained these high levels from positive performance KR but reported lower SE after exposure to poor performance KR. These results support the OPTIMAL theory of motor learning, positing that enhanced expectancies can facilitate motor learning (Wulf \& Lewthwaite, 2016). Practical implications of these results are the particular benefits of positive performance KR for novices with low self-expectancies and the beneficial maintenance effects of high SE from positive performance KR for professional athletes. A specific focus of future research might be to investigate the relationship between SE and positive performance KR on the motor performance of professional athletes. In addition, future research might investigate the effects of various other types of feedback (e.g., absolute vs. comparative feedback, KR vs. KP) on different types of 
motivational states (e.g., intrinsic/extrinsic motivation, goal orientation) in order to gain further insights into the complex relationships between feedback and individual motivation characteristics on motor performance and learning.

\section{Acknowledgments}

The authors thank all the students who participated in this study.

\section{Declaration of Conflicting Interests}

The author(s) declared no potential conflicts of interest with respect to the research, authorship, and/or publication of this article.

\section{Funding}

The author(s) received no financial support for the research, authorship, and/or publication of this article.

\section{ORCID iD}

Saeed Ghorbani (D) https://orcid.org/0000-0001-5670-8030

\section{References}

Ávila, L. T. G., Chiviacowsky, S., Wulf, G., \& Lewthwaite, R. (2012). Positive socialcomparative feedback enhances motor learning in children. Psychology of Sport and Exercise, 13, 849-853.

Badami, R., VaezMousavi, M., Wulf, G., \& Namazizadeh, M. (2011). Feedback after good trials enhances intrinsic motivation. Research Quarterly for Exercise and Sport, $82,360-364$.

Badami, R., VaezMousavi, M., Wulf, G., \& Namazizadeh, M. (2012). Feedback about more accurate versus less accurate trials: Differential effects on self-confidence and activation. Research Quarterly for Exercise and Sport, 83, 196-203.

Bandura, A. (1977). Self-efficacy: Toward a unifying theory of behavioral change. Psychological Review. 84(2), 191-215.

Chiviacowsky, S., \& Wulf, G. (2007). Feedback after good trials enhances learning. Research Quarterly for Exercise and Sport, 78, 40-47.

Chiviacowsky, S., Wulf, G., Wally, R., \& Borges, T. (2009). KR after good trials enhances learning in older adults. Research Quarterly for Exercise and Sport, 80, 663-668.

Ghorbani, S. (2019). Motivational effects of enhancing expectancies and autonomy for motor learning: An examination of the OPTIMAL theory. The Journal of General Psychology, 146(1), 79-92.

Gonçalves, G. S., Cardozo, P. L., Valentini, N. C., \& Chiviacowsky, S. (2018). Enhancing performance expectancies through positive comparative feedback facilitates the learning of basketball free throw in children. Psychology of Sport \& Exercise, 36, 174-177.

Grand, K. F., Daou, M., Lohse, K. R., \& Miller, M. W. (2017). Investigating the mechanisms underlying the effects of an incidental choice on motor learning. Journal of Motor Learning and Development, 5(2), 207-226. 
Lessa, H. T., Tani, G., \& Chiviacowsky, S. (2018). Benefits of enhanced expectancies through temporal-comparative feedback for motor learning in older adults. International Journal of Sport Psychology, 49, 521-530.

Montes, J., Wulf, G., \& Navalta, J. W. (2018). Maximal aerobic capacity can be increased by enhancing performers' expectancies. The Journal of Sports Medicine and Physical Fitness, 58(5), 744-749.

Ong, N. T., \& Hodges, N. J. (2018). Balancing our perceptions of the efficacy of successbased feedback manipulations on motor learning. Journal of Motor Behavior, 50(6), 614-630.

Pascua, L. A., Wulf, G., \& Lewthwaite, R. (2015). Additive benefits of external focus and enhanced performance expectancy for motor learning. Journal of Sports Sciences, 33(1), 58-66.

Saemi, E., Porter, J. M., Ghotbi-Varzaneh, A., Zarghami, M., \& Maleki, F. (2012). Knowledge of results after relatively good trials enhances self-efficacy and motor learning. Psychology of Sport and Exercise, 13, 378-382.

Saemi, E., Wulf, G., Ghotbi-Varzaneh, A., \& Zarghami, M. (2011). Feedback after good versus poor trials enhances motor learning in children. Revista Brasileira de Educaçao Fisica e Esporte (Brazilian Journal of Physical Education and Sport), 25(4), 673-681.

Stoate, I., Wulf, G., \& Lewthwaite, R. (2012). Enhanced expectancies improve movement efficiency in runners. Journal of Sports Sciences, 30, 815-823.

Wulf, G., Chiviacowsky, S., \& Cardozo, P. (2014). Additive benefits of autonomy support and enhanced expectancies for motor learning. Human Movement Science, 37, $12-20$.

Wulf, G., \& Lewthwaite, R. (2016). Optimizing performance through intrinsic motivation and attention for learning: The OPTIMAL theory of motor learning. Psychonomic Bulletin \& Review, 23, 1382-1414.

\section{Author Biographies}

Saeed Ghorbani (PhD) is an assistant professor for Motor Control and Learning at the Islamic Azad University, Aliabad Katoul Branch, Iran. His research focuses on various variables affecting motor learning such as action observation, feedback, etc.

Andreas Bund ( $\mathrm{PhD})$ is an associate professor for Physical Education, Motor Learning, and Development at the University of Luxembourg, Luxembourg. He is in charge of the formation of physical education teachers for primary schools. His research is focused on motor performance, motor learning and motor development in school settings, e.g., the implementation and evaluation of motor tests, observational and self-regulated motor skill learning and the measurement of physical activity levels of children. 